\title{
Curriculum für die Facharztausbildung und die Weiterbildung in der Muskuloskelettalen Radiologie
}

\author{
Autoren \\ Rainer Braunschweig1, Rolf Janka², Marc Regier ${ }^{3}$ \\ Vorstand der AG Bildgebende Verfahren des Bewegungsapparates in der DRG \\ Institute \\ 1 Von Wedderkopstraße 2-4, 23847 Steinhorst \\ 2 Radiologisches Institut, Universitätsklinikum Erlangen, \\ Maximiliansplatz 3, 91054 Erlangen \\ 3 Radiologie München, Burgstraße 7, 80331 München \\ Bibliografie \\ DOI https://doi.org/10.1055/a-1091-4072 \\ Online-Publikation: 2020 \\ Fortschr Röntgenstr 2020; 192: 408-417 \\ (c) Georg Thieme Verlag KG, Stuttgart · New York \\ ISSN 1438-9029 \\ Korrespondenzadresse \\ Prof. Marc Regier \\ Radiologie München, Burgstraße 7, 80331 München, \\ Germany \\ Tel.: ++49/89/212196426 \\ m.regier@radiologie-muenchen.de
}

\section{Begleitwort}

Die AG Bildgebende Verfahren des Bewegungsapparates (AG BVB) in der Deutschen Röntgengesellschaft (DRG) stellt ein umfassendes und präzisiertes Facharzt- und Weiterbildungs-Curriculum vor - Warum?

Die Radiologie ist ein klinisches Fachgebiet, das seine Attraktivität aus der fachspezifischen Kombination zweier ärztlicher Aufgaben bezieht: Patientenorientierung und klinische Interdisziplinarität.

Diese begründen und sichern den hohen klinischen und damit auch wirtschaftlichen Wert der Bildgebung in z. B. evidenz- und leitlinienbasierten Versorgungskonzepten.

Der Mediziner/der Radiologe wird zum Arzt durch die Wahrnehmung seiner klinischen Aufgabe - d. h. durch den Patienten.

Um dieser Versorgungsaufgabe gerecht zu werden, sind grundsätzlich 3 akademische Umsetzungsebenen nötig:

- wissenschaftliche Erkenntnis (Forschung),

- Wissensvermittlung (Lehre) und

- klinische Anwendung (Patientenversorgung).

Die Wissensanwendung durch praktische Fähigkeiten und die kollegial-interdisziplinäre Zusammenarbeit sind die wesentlichen Voraussetzungen. Die Ausbildung von Ärzten/-innen dient vor allem der Sicherstellung der Gesundheitsversorgung der Bevölkerung.

„Bilder sind Dokumente des Augenscheins“ („Radiologische Fachgutachten“; Hrsg. F.H.W. Heuck, Springer 1998) - diese

Das Begleitwort zum Curriculum für die Facharztausbildung und die Weiterbildung in der Muskuloskelettalen Radiologie wird parallel in der Zeitschrift Der Radiologe, 5/2020 (DOI 10.1007/s00117-020-00680-8) publiziert. grundlegende Funktion der Bildgebenden Diagnostik ist der Kern-Beitrag der Radiologie in Kliniken und Praxen.

In der Radiologie umfasst daher die Ausbildungsverantwortung gegenüber den Patienten,

- die subtile Kenntnis der bildgebenden Verfahren, insbesondere

- deren leistungsadäquate und patienten- und damit fragestellungsorientierte Indikation (Strahlenschutzgesetz und -verordnung) und

- deren evidenzbasierte Durchführung und Beurteilung.

Um dies sicherzustellen hat die Musterweiterbildungsordnung das Fachgebiet der Radiologie dankenswerterweise grundlegend gestärkt und die DRG die fachliche Aus-, Fort- und Weiterbildung im Sinne der konsequenten Qualitätssicherung in allen Teilbereichen der Bildgebenden Diagnostik aktuell und umfassend präzisiert.

Neben der Koordination und Präsentation nationaler und internationaler wissenschaftlicher Aktivitäten stehen eine Vielzahl strukturierter Weiterbildungsangebote für alle Ausbildungsebenen in unterschiedlichen Formaten zur Verfügung.

Die AG Bildgebende Verfahren des Bewegungsapparates hat vor diesem Hintergrund ein sowohl national orientiertes als auch international basiertes Curriculum zur Facharztausbildung entwickelt und mit weiterführenden Spezialisierungen und Zertifikaten ergänzt. Damit trägt die Deutsche Röntgengesellschaft ihrem Anspruch Rechnung, das gesamte Fachgebiet umfassend klinisch zu vertreten, und schafft inhaltliche Voraussetzungen für die adäquate wissensbasierte Versorgung unserer Patienten.

Nachfolgend stellt die AG Bildgebende Verfahren des Bewegungsapparates

- Aus- und Weiterbildungsinhalte vor,

- erläutert deren Inhalte und Zielsetzung und 
- bindet diese in den umfassenden Kanon der bildgebenden Verfahren aller Organsysteme (DRG) ein.

Besonderer Wert wird neben den Inhalten auf ein zueinander komplementäres klinisches Aus- und Weiterbildungssystem aus

- ärztlich-klinischer Tätigkeit,

- Kursen und Kongressbesuchen sowie

- digitalen Weiterbildungs- und interaktiven Prüfungsformaten

gelegt. Digitale Repetitionssysteme („Akademie für Fort- und Weiterbildung in der Radiologie“/"conrad“) sind originärer Bestandteil des Trainingsangebots.

Zusammenfassend stellt das hier vorgestellte MSK-Curriculum die klinischen Grundlagen für eine gleichermaßen umfassende wie spezialisierte Aus- und Weiterbildung sicher.

Dabei sind fachliches Wissen und dessen klinische Anwendung und die wissenschaftliche Weiterentwicklung Bestandteil des Ausund Weiterbildungsangebots und stellen sich den klinischen Herausforderungen an das Fachgebiet. Die klinisch notwendige ganzheitliche Vertretung der Bildgebenden Diagnostik in der Radiologie wird damit sowohl gegenüber den klinischen Kollegen/-innen als auch gegenüber den Patienten/-innen sichergestellt.

\section{Begriffsdefinitionen:}

- Inhalte:

- „Kenntnisse“: abrufbares Wissen (Standard- und Spezialliteratur, klinische Erfahrung, Wissensinhalte aus den Weiterbildungseinrichtungen)

- „Verständnis“: prinzipielle Einordnung von Zusammenhängen (praktische Erfahrung, Studienwissen, Literatur)

- „Fähigkeiten und Fertigkeiten“: praktische, ärztlich-klinische Erfahrungen (belegbare Zahlen von durchgeführten Injektionen, Interventionen, Durchleuchtungen und z. B. Notfallsituationen, interdisziplinäre, kollegiale und patientenorientierte Kommunikation)

- Graduierungen (in absteigender Wichtung)

- „komplett“: in der Literatur verfügbare Wissensinhalte

- „umfassend“:klinisch relevante Wissensinhalte

- „grundlegend“: prinzipielle Wissensinhalte

\section{Inhaltsverzeichnis}

\begin{tabular}{|l|l|l|}
\hline I & Lerninhalte Facharztstandard MSK-Radiologie & 409 \\
\hline 1.1 & Allgemein & 409 \\
\hline 1.2 & Wissen & 410 \\
\hline I.2.1 & Traumata - akut und Verlaufskontrollen & 410 \\
\hline I.2.2 & Infektionen & 410 \\
\hline I.2.3 & Tumoren und tumor-like lesions & 410 \\
\hline I.2.4 & Hämatologische Erkrankungen & 410 \\
\hline I.2.5 & Metabolische, endokrine und toxische & 410 \\
\hline I.2.6 & Erkrankungen & \\
\hline I.2.7 & Gelenke & 411 \\
\hline I.2.8 & Kongenitale und Entwicklungsstörungen & 411 \\
\hline & & 411 \\
\hline
\end{tabular}

\section{Inhaltsverzeichnis}

\begin{tabular}{|c|c|c|}
\hline 1.3 & KOMPETENZEN (Fertigkeiten und Fähigkeiten) & 411 \\
\hline 1.3.1 & Technisch-klinische Anwendungen & 411 \\
\hline 1.3.2 & Klinisch-radiologische Tätigkeiten & 411 \\
\hline ॥ & Lerninhalte Q1 Spezialwissen MSK-Radiologie & 412 \\
\hline II. 1 & Allgemein & 412 \\
\hline 11.2 & Wissen & 412 \\
\hline 11.2 .1 & Traumata - akut und Verlaufskontrolle & 412 \\
\hline 11.2 .2 & Infektionen & 412 \\
\hline 11.2 .3 & Tumoren und tumor-like lesions & 413 \\
\hline II.2.4 & Hämatologische Erkrankungen & 413 \\
\hline 11.2 .5 & $\begin{array}{l}\text { Metabolische, endokrine und toxische } \\
\text { Erkrankungen }\end{array}$ & 413 \\
\hline 11.2 .6 & Gelenke & 413 \\
\hline 11.2 .7 & Kongenitale und Entwicklungsstörungen & 413 \\
\hline 11.2 .8 & Verschiedenes & 413 \\
\hline 11.3 & KOMPETENZEN (Fertigkeiten und Fähigkeiten) & 413 \\
\hline II.3.1 & Technisch-klinische Anwendungen & 413 \\
\hline 11.3 .2 & Klinisch-radiologische Tätigkeiten & 414 \\
\hline III & Lerninhalte Q2 Expertenwissen MSK-Radiologie & 414 \\
\hline III. 1 & Allgemein & 414 \\
\hline III. 2 & Wissen & 415 \\
\hline III.2.1 & Traumata - akut und Verlaufskontrolle & 415 \\
\hline III.2.2 & Infektionen & 415 \\
\hline III.2.3 & Tumoren und tumor-like lesions & 415 \\
\hline III.2.4 & Hämatologische Erkrankungen & 415 \\
\hline III. 2.5 & $\begin{array}{l}\text { Metabolische, endokrine und toxische } \\
\text { Erkrankungen }\end{array}$ & 416 \\
\hline III.2.6 & Gelenke & 416 \\
\hline III.2.7 & Kongenitale und Entwicklungsstörungen & 416 \\
\hline III.2.8 & Verschiedenes & 416 \\
\hline III.3 & KOMPETENZEN (Fertigkeiten und Fähigkeiten) & 416 \\
\hline III.3.1 & Technisch-klinische Anwendungen & 416 \\
\hline 111.3.2 & Klinisch-radiologische Tätigkeiten & 417 \\
\hline
\end{tabular}

\section{Lerninhalte Facharztstandard MSK-Radiologie (5 Jahre/60 Monate)}

\section{I.1 Allgemein}

- Umfassende Kenntnisse der Anatomie aller für die Bildgebung des Bewegungsapparates relevanten Strukturen

- Grundlegende Kenntnisse über normale Skelettvarianten, die Krankheiten imitieren können (z. B. Sesambeine oder „Schaltknochen“ vs. Frakturen)

- Grundlegendes Verständnis der Stufen der „Skelettentwicklung“"

- Grundlegende Kenntnisse der Embryologie des Skeletts 
- Komplette Kenntnisse der klinischen Indikationen, Kontraindikationen und potenziellen Gefahren (z. B. Strahlenbelastung, Verfahrensbelastung) der bildgebenden Verfahren bei muskuloskelettalen Erkrankungen und bei Traumata

- Grundlegende Kenntnisse klinischer, insbesondere chirurgischer und internistischer, pathologischer und pathophysiologischer Zusammenhänge des Bewegungsapparates

- Umfassende Kenntnisse der Bildgebungsmerkmale (lexikalisierte Befundkriterien) und klinischen Manifestationen von Erkrankungen und Traumata des Bewegungsapparates in der Projektionsradiografie, CT, MRT, Durchleuchtung (z. B. Arthrografie und Funktionsanalysen) und im Ultraschall

- Grundlegende Kenntnisse der Differenzialdiagnosen, die für das klinische und bildgebende Erscheinungsbild von Erkrankungen oder Traumata des Bewegungsapparates relevant sind

\section{I.2 Wissen}

\section{I.2.1 Traumata - akut und Verlaufskontrollen}

- Grundlegende Kenntnisse der Klassifizierungen von Frakturen und Luxationen, z. B. systematische Grundlagen der Outcome-, morphologisch- oder operativ-orientierten (z. B. AO- oder organspezifisch-operativ orientierte (z. B. Weberoder Pauwels-)) oder Verletzungsmechanismus- bzw. funktionell-orientierte Klassifikationen (z. B. Essex-Lopresti- oder Maisonneuve-Fraktur)

- Umfassende Kenntnisse typischer und lexikalisierter Bildkriterien und grundlegende klinische Kenntnisse von:

- Frakturen und Luxationen im Erwachsenenskelett (ab 14 Jahre/s. I.2.1.)

- der Skelettentwicklung

- chondralen und osteochondralen Läsionen (z. B. Osteochondrose dissecans vs. flake-fracture)

- Konsolidierungsprozessen und Komplikationen bei Frakturen und Luxationen (z. B. verzögerte Konsolidierung, Pseudarthrose, avaskuläre Nekrose, Reflexdystrophie, komplexes und/ oder regionales Schmerzsyndrom, Myositis ossificans etc.)

\section{I.2.1.1 Frakturen und Luxationen im Einzelnen als Schwerpunktauflistung}

- Stressfrakturen, einschließlich Ermüdungs- und Insuffizienzbrüchen

- Schädel- und Gesichtsschädelfrakturen

- (Ausriss-) Avulsionsfrakturen

- pathologische, nichttraumatische Frakturen

- Frakturen der Wirbelsäule, einschließlich der Spondylolyse

- Frakturen und Luxationen der Schulter (Klavikulafrakturen, Skapulafrakturen und Schulterluxation/, ,habituelle“ Instabilitäten)

- Frakturen und Luxationen der oberen Gliedmaßen (z. B. Humerusfrakturen, Ellenbogenfrakturen und -luxationen, proximale und distale Unterarmfrakturen und -luxationen, Handgelenkfrakturen/-luxationen und Handfrakturen und -luxationen)

- Frakturen und Luxationen des Beckens einschließlich Weichteilverletzungen

- Frakturen und Luxationen der unteren Extremitäten (z. B. Hüftfrakturen und -luxationen, Femurfrakturen, Tibia- und Fibulafrak- turen (einschließlich des Sprunggelenks), Rückfußfrakturen, Tarsometatarsalfrakturen und -luxationen sowie Vorfußfrakturen und -luxationen)

- Verletzungen oder Gefügestörungen

- des Schultergelenks (z. B. der Rotatorenmanschette, des Glenoid-Labrum-Komplexes und der Bizepssehne)

- des Handgelenks (z. B. des Discus triangularis und der interkarpalen Bänder)

- des Hüftgelenks (z. B. femoroacetabuläres Impingement)

- des Knies (z. B. Meniskus, Kreuzband- und Kollateralbänder)

- des Sprunggelenks (z. B. der Hauptsehnen und Bänder)

\section{I.2.2 Infektionen}

- Grundlegende Kenntnisse typischer Bildkriterien und grundlegende Kenntnisse klinischer Merkmale

- der akuten, subakuten und chronischen hämatogenen Osteomyelitis

- der posttraumatischen und postoperativen (Implantat-) Osteomyelitis

\section{I.2.3 Tumoren und tumor-like lesions}

- Grundlegende Kenntnisse der typischen Bildkriterien, Prinzipien der Tumorcharakterisierung und des Stagings (z. B. klinisch, pathologisch-anatomisch/histologisch)

- von knochenbildenden Tumoren (z. B. Osteom, Osteoidosteom, Osteoblastom, Osteosarkom (typische Formen und Varianten)

- von knorpelbildenden Tumoren (z. B. Osteochondrom, Enchondrom, Chondroblastom, Chondrosarkom (zentral und peripher)

- von fibrösen Tumoren (z. B. fibröser Kortikalisdefekt, nicht ossifizierendes Fibrom, fibröse Dysplasie)

- von hämatopoetischen und retikuloendothelialen Tumoren (z. B. Riesenzelltumor, Langerhans-Zellhistiozytose, maligne Rundzelltumoren (Ewing-Sarkom, Lymphom und Leukosen, Myelom, Plasmozytom)

- einfacher und aneurysmatischer Knochenzysten

- von Metastasen

- von „dont't touch lesions“

- von Tumoren des Fettgewebes (z. B. Lipom und Liposarkom)

- von Tumoren neuronalen Ursprungs (z. B. Neurofibrom und Schwannom)

- von Tumoren vaskulären Ursprungs (z. B. Hämangiom)

- von Weichteilsarkomen

\section{I.2.4 Hämatologische Erkrankungen}

- Grundlegende Kenntnisse der Bildkriterien und der klinischen Merkmale der Myelofibrose

\section{I.2.5 Metabolische, endokrine und toxische Erkrankungen}

- Grundlegende Kenntnisse der Bildkriterien und klinischen Merkmale

- der Osteomalazie

- des primären, sekundären und tertiären Hyperparathyreoidismus (einschließlich chronischer Niereninsuffizienz) 
- der Osteoporose (einschließlich grundlegender Konzepte der Knochenbildgebung und Knochendichtemessung)

I.2.6 Gelenke

- Umfassende Kenntnisse der Bildkriterien und klinischer Merkmale

- degenerativer Erkrankungen der Wirbelsäulen-, Bandscheiben- und Facettengelenke, der Becken- und peripherer Gelenke

- entzündlicher Erkrankungen (z. B. rheumatoide Arthritis, juvenile rheumatoide Arthritis, Spondylitis ankylosans, psoriatische Arthritis, enteropathische Arthropathien und infektiöse Arthritis (pyogen und tuberkulös), seronegative Arthritidien)

- von Kristallarthropathien (Pyrophosphat-Arthropathie, Hydroxyapatit-Ablagerungskrankheit und Gicht)

- Grundlegende Kenntnisse der Bildkriterien und klinischer Merkmale

- von tumorartigen Gelenkveränderungen (z. B. Ganglion, synoviale Chondromatose, Morton-Neurom und pigmentierte villonoduläre Synovitis)

- bei Neuropathien (diabetischer Fuß, Charcot-Gelenke und Pseudo-Charcot-Gelenk (steroidinduziert))

- in der Endo- und Revisionsprothetik (Hüfte und Knie)

\section{I.2.7 Kongenitale und Entwicklungsstörungen}

\section{- Grundlegende Kenntnisse der Bildkriterien und klinischer} Merkmale

- angeborener Erkrankungen der Wirbelsäule (z. B. Skoliose (kongenitale und idiopathische) und Dysraphien (inkomplett und komplett))

- angeborener Hand- und Handgelenkstörungen (z. B. Madelung-Deformität (idiopathische und andere Ursachen))

- von Erkrankungen der Hüfte (z. B. Hüftdysplasie, M. Perthes, Epiphysiolyse)

- der angeborenen Koalitionen (z. B. Tarsalkoalition)

- angeborener Knochenentwicklungsstörungen (z. B. Achondroplasie und osteogenesis imperfecta)

- angeborener Knochendichtestörungen (z. B. Osteopetrose, Melorheostose und Osteopoikilie)

- tumorähnlicher Läsionen (z. B. Exostosen/Osteochondrome) und M. Ollier)

\section{I.2.8 Verschiedenes}

- Umfassende Kenntnisse der Bildkriterien und grundlegende Kenntnisse klinischer Merkmale

- des M. Paget

- der transienten Osteoporose/-penie

- der Osteonekrosen (aseptische, traumatische etc.)

- der Weichteilverkalkungen/Weichteilossifikation

\section{I.3 KOMPETENZEN (Fertigkeiten und Fähigkeiten)}

\section{I.3.1 Technisch-klinische Anwendungen}

- Auswahl der geeignetsten Bildgebungsmethode des Bewegungsapparates abhängig vom klinischen Problem (klinische Fragestellung/rechtfertigende Indikation)
- Auswahl des am besten geeigneten Kontrastmittels und seine optimale Verwendung (Dosierung, Flow-Protokoll) entsprechend der Bildgebungstechnik und der klinischen Fragestellung bei der Bildgebung des Bewegungsapparates

- Durchführung dynamischer Funktionsstudien der Wirbelsäule und der Gelenke

- Grundlagen der Einstellungstechniken der Projektionsradiografie des Stammskeletts und der Extremitäten einschließlich aller Spezialeinstellungen

- Wissen über die Durchführung einfacher bildgesteuerter, perkutaner Biopsien, Drainagen und Schmerztherapien des Bewegungsapparates (Durchführung von komplexen bildgesteuerten Biopsien, Drainagen und Schmerztherapien (z. B. GanglionBlockaden) des Bewegungsapparates unter Aufsicht)

- Planung einer CT-Untersuchung des Bewegungsapparates be individueller klinischer Situation und Fragestellung (individuelle Anwendung, keine standardisierte Protokollsituation - z. B. Notfall - Beachtung des ALARA-Prinzips)

- Planung einer MRT-Untersuchung des Bewegungsapparates bei individueller klinischer Situation und Fragestellung im Hinblick auf räumliche Auflösung, Wahl der Sequenzen und die mögliche Verwendung intravenöser und/oder intrakavitärer Kontrastmittel (z. B. direkte vs. indirekter Arthrografie)

- Grundlegende Kenntnisse über die Durchführung von Kontrastmittelinjektionen in Gelenke (z. B. Hüfte, Schulter und Handgelenk (z. B. MR-, CT-Arthrografie))

- Sichere Anwendung von Nachbearbeitungsalgorithmen für Bildgebungsstudien im Muskel- und Skelettbereich, einschließlich multiplanarer Reformation (MPR), Maximalintensitätsprojektion (MIP), Minimalintensitätsprojektion (MinIP) und Fusion von Bildern oder Bildstudien

\section{I.3.2 Klinisch-radiologische Tätigkeiten}

- Umfassende Kenntnisse zur Indikationsstellung diagnostischer und interventioneller Verfahren des Bewegungsapparates

- Umfassende Kenntnisse zur Moderation unterschiedlicher bildgebender Verfahren zur Beurteilung von Erkrankungen des Bewegungsapparates

- Umfassende Kommunikation mit dem Patienten zur Aufklärung, Beratung und Erlangung der Einverständniserklärung vor der diagnostischen Bildgebung oder vor interventionellen Verfahren des Bewegungsapparates sowie bei der Befunderläuterung (inkl. Angehöriger)

- Komplette Kenntnisse zur optimalen Wahl der Bildgebungsparameter für Röntgen- (ALARA-Prinzip/Strahlenschutzgesetzgebung), Ultraschall-, CT- (ALARA-Prinzip/Strahlenschutzgesetzgebung) und MRT-Untersuchungen des Bewegungsapparates

- Überwachung und Unterweisung des technischen Personals zur Sicherstellung geeigneter Untersuchungsdokumente (hard- und soft-copy, DIN-Normierungen) des Bewegungsapparates

- Sichere Beherrschung der Qualitätskontrollvorgaben (DINNormen 6868, Leitlinien der Bundesärztekammer in jeweils 
aktueller Fassung) von Bildgebungsuntersuchungen des Bewegungsapparates

- Komplette Beherrschung der Befundung und Beurteilung der relevanten bildgebenden Verfahren (Projektionsradiografie, $\mathrm{CT}, \mathrm{MR}$, Durchleuchtung, DSA und in Grundsätzen des Ultraschalls) des Bewegungsapparates unter Verwendung standardisierter Befundvorgaben (z. B. strukturierte Befundung, lexikalisierte Bildkriterien, Befundaufbau - DIN-Normen)

- Grundlegende Fähigkeiten und Fertigkeiten zur klinischen Untersuchung des Bewegungsapparates (s. auch „rechtfertigende Indikation“/Strahlenschutzgesetzgebung)

- Grundlegende Kenntnisse in der Befundung des Bewegungsapparates nach internationalen Standards (RECIST, WHO)

- Sichere Erkennung von Notfällen

- Erfahrungen in der interdisziplinären Befunddiskussion und in der kollegialen Fallbesprechung

\section{Lerninhalte Q1 Spezialwissen MSK-Radiologie}

\section{II.1 Allgemein}

- Komplette Kenntnisse der Anatomie aller für die Bildgebung des Bewegungsapparates relevanten Strukturen

- Umfassende Kenntnisse über normale Skelettvarianten, die Krankheiten imitieren können (z. B. Sesambeine oder „Schaltknochen“ vs. Frakturen)

- Umfassendes Verständnis der Stufen der „Skelettentwicklung“

- Grundlegende Kenntnisse der Embryologie des Skeletts

- Komplette Kenntnisse der klinischen Indikationen, Kontraindikationen und potenziellen Gefahren (z. B. Strahlenbelastung, Verfahrensbelastung) der bildgebenden Verfahren bei muskuloskelettalen Erkrankungen und bei Traumata

- Umfassende Kenntnisse klinischer, insbesondere chirurgischer und internistischer, pathologischer und pathophysiologischer Zusammenhänge des Bewegungsapparates

- Umfassende Kenntnisse der Bildgebungsmerkmale (lexikalisierte Befundkriterien) und klinischen Manifestationen von Erkrankungen und Traumata des Bewegungsapparates in der Projektionsradiografie, CT, MRT, Durchleuchtung (z. B. Arthrografie und Funktionsanalysen) und im Ultraschall

- Umfassende Kenntnisse der Differenzialdiagnosen, die für das klinische und bildgebende Erscheinungsbild von Erkrankungen oder Traumata des Bewegungsapparates relevant sind

\section{II.2 Wissen}

\section{II.2.1 Traumata - akut und Verlaufskontrolle}

- Umfassende Kenntnisse der Klassifizierungen von Frakturen und Luxationen, z. B. systematische Grundlagen der Outcome-, morphologisch- oder operativ-orientierten (z. B. AO- oder organspezifisch-operativ orientierte (z. B. Weber- oder Pauwels-)) oder Verletzungsmechanismus- bzw. funktionell-orientierten (z. B. Essex-Lopresti- oder Maisonneuve-Fraktur) Klassifikationen
- Umfassende Kenntnisse typischer und lexikalisierter Bildkriterien und grundlegende klinische Kenntnisse von:

- Frakturen und Luxationen im Erwachsenenskelett (ab 14 Jahre/s. I.2.1.)

- der Skelettentwicklung

- chondralen und osteochondralen Läsionen (z. B. Osteochondrose dissecans vs. flake-fracture)

- Konsolidierungsprozessen und Komplikationen bei Frakturen und Luxationen (z. B. verzögerte Konsolidierung, Pseudarthrose, avaskuläre Nekrose, Reflexdystrophie, komplexes und/ oder regionales Schmerzsyndrom, Myositis ossificans etc.)

\section{II.2.1.1 Frakturen und Luxationen im Einzelnen als Schwerpunktauflistung}

- Stressfrakturen, einschließlich Ermüdungs- und Insuffizienzbrüchen

- Schädel- und Gesichtsschädelfrakturen

- (Ausriss-) Avulsionsfrakturen und Insertionstendopathien

- pathologische, nichttraumatische Frakturen

- Frakturen der Wirbelsäule, einschließlich der Spondylolyse

- Frakturen und Luxationen der Schulter (z. B. sternoklavikuläre und akromioklavikuläre Luxationen, Klavikulafrakturen, Skapulafrakturen und Schulterluxation/,habituelle“ Instabilitäten)

- Frakturen und Luxationen der oberen Gliedmaßen (z. B. Humerusfrakturen, Ellenbogenfrakturen und -luxationen, proximale und distale Unterarmfrakturen und -luxationen, Handgelenkfrakturen/-luxationen und Handfrakturen und -luxationen)

- Frakturen und Luxationen des Beckens einschließlich Weichteilverletzungen

- Frakturen und Luxationen der unteren Extremitäten (z. B. Hüftfrakturen und -luxationen, Femurfrakturen, Tibia- und Fibulafrakturen (einschließlich des Sprunggelenks), Rückfußfrakturen, Tarsometatarsalfrakturen und -luxationen sowie Vorfußfrakturen und -luxationen)

- Verletzungen oder Gefügestörungen

- des Schultergelenks (z. B. der Rotatorenmanschette, des Glenoid-Labrum-Komplexes und der Bizepssehne)

- des Ellbogengelenks

- des Handgelenks (z. B. des Discus triangularis und der interkarpalen Bänder)

- der Finger

- des Hüftgelenks (z. B. Labrum und FAl)

- des Knies (z. B. der Menisken, Kreuz- und Kollateralbänder und der posterolateralen Ecke)

- des Sprunggelenks (z. B. der Hauptsehnen, des Innen- und Außenbandapparates und des Tibiospringligaments)

- der Fußwurzel- und Zehengelenke

\section{II.2.2 Infektionen}

- Umfassende Kenntnisse typischer Bildkriterien und grundlegende Kenntnisse klinischer Merkmale

- der akuten, subakuten und chronischen hämatogenen Osteomyelitis

- der posttraumatischen und postoperativen (Implantat-) Osteomyelitis

- der Tuberkulose des Bewegungsapparates 


\section{II.2.3 Tumoren und tumor-like lesions}

- Umfassende Kenntnisse der typischen Bildkriterien, Prinzipien der Tumorcharakterisierung und des Stagings (z. B. klinisch, pathologisch-anatomisch/histologisch)

- von knochenbildenden Tumoren (z. B. Osteom, Osteoidosteom, Osteoblastom, Osteosarkom (typische Formen und Varianten))

- von knorpelbildenden Tumoren (z. B. Osteochondrom, Enchondrom, Chondroblastom, Chondromyxoid-Fibrom und Chondrosarkom (zentral und peripher))

- von fibrösen Tumoren (z. B. fibröser Kortikalisdefekt, nicht ossifizierendes Fibrom, fibröse Dysplasie, Fibrosarkom, malignes fibröses Histiozytom)

- von hämatopoetischen und retikuloendothelialen Tumoren (z. B. Riesenzelltumor, Langerhans-Zellhistiozytose, maligne Rundzelltumoren (Ewing-Sarkom, Lymphom und Leukosen), Myelom, Plasmozytom)

- einfacher und aneurysmatischer Knochenzysten

- von Metastasen

- von „dont't touch lesions“

- von Tumoren des Fettgewebes (z. B. Lipom und Liposarkom)

- von Tumoren neuronalen Ursprungs (z. B. Neurofibrom und Schwannom)

- von Tumoren vaskulären Ursprungs (z. B. Hämangiom)

- von Weichteilsarkomen

\section{II.2.4 Hämatologische Erkrankungen}

- Grundlegende Kenntnisse der Bildkriterien und der klinischen Merkmale von Hämoglobinopathien (z. B. Sichelzellenanämie und Thalassämie)

- Grundlegende Kenntnisse der Bildkriterien und der klinischen Merkmale der Myelofibrose

\section{II.2.5 Metabolische, endokrine und toxische Erkrankungen}

- Grundlegende Kenntnisse der Bildkriterien und der klinischen Merkmale

- der Rachitis und Osteomalazie

- des primären, sekundären und tertiären Hyperparathyreoidismus (einschließlich chronischer Niereninsuffizienz)

- der Osteoporose (einschließlich grundlegender Konzepte der Knochenbildgebung und Knochendichtemessung)

- der Fluorose und sonstiger Mineralosen

\section{II.2.6 Gelenke}

\section{- Umfassende Kenntnisse der Bildkriterien und umfassende} Kenntnisse der klinischen Merkmale

- degenerativer Erkrankungen der Wirbelsäulen-, Bandscheiben- und Facettengelenke, der Becken- und peripherer Gelenke

- entzündlicher Erkrankungen (z. B. rheumatoide Arthritis, juvenile rheumatoide Arthritis, Spondylitis ankylosans, psoriatische Arthritis, enteropathische Arthropathien und infektiöse Arthritis (pyogen und tuberkulös), seronegative Arthritidien)

- von Kristallarthropathien (Pyrophosphat-Arthropathie, Hydroxyapatit-Ablagerungskrankheit und Gicht)
- von tumorartigen Gelenkveränderungen (z. B. Ganglion, synoviale Chondromatose, Morton-Neurom und pigmentierte villonoduläre Synovitis)

- bei Neuropathien (diabetischer Fuß, Charcot-Gelenke und Pseudo-Charcot-Gelenk (steroidinduziert))

- in der Endo- und Revisionsprothetik (Hüfte und Knie)

\section{II.2.7 Kongenitale und Entwicklungsstörungen}

- Grundlegende Kenntnisse der Bildkriterien und der klinischen Merkmale

- angeborener Erkrankungen der Wirbelsäule (z. B. Skoliose (kongenitale und idiopathische) und Dysraphien (inkomplett und komplett))

- angeborener Erkrankungen der Schulter (z. B. Sprengels-Deformität)

- angeborener Hand- und Handgelenkstörungen (z. B. Madelung-Deformität (idiopathische und andere Ursachen))

- von Erkrankungen der Hüfte (z. B. Hüftdysplasie, M. Perthes, Epiphysiolyse, femoroacetabuläres Impingement)

- der angeborenen Tarsalkoalition

- von Knochendysplasien (z. B. Dysmelien, Dysraphien, epiund/oder metaphysäre Dysplasien etc. - s. „Orpha-Klassifikation")

- bei multipler epiphysealer Dysplasie

- angeborener Knochenentwicklungsstörungen (z. B. Achondroplasie und osteogenesis imperfecta)

- angeborener Knochendichtestörungen (z. B. Osteopetrose, Melorheostose und Osteopoikilie)

- tumorähnlicher Läsionen (z. B. Exostosen/Osteochondrome und M. Ollier)

- der muskuloskelettalen Manifestationen der Neurofibromatose

\section{II.2.8 Verschiedenes}

- Umfassende Kenntnisse der Bildkriterien und grundlegende Kenntnisse der klinischen Merkmale

- des M. Paget

- der Sarkoidose (z. B. M. jüngling)

- der Varianten der hypertrophen Osteoarthropathien (seropositiv und -negativ)

- der transienten Osteoporose/-penie

- der Osteonekrosen (aseptische, traumatische etc.)

- der Weichteilverkalkungen/Weichteilossifikation

\section{II.3 KOMPETENZEN (Fertigkeiten und Fähigkeiten)}

\section{II.3.1 Technisch-klinische Anwendungen}

- Auswahl der geeignetsten Bildgebungsmethode des Bewegungsapparates abhängig vom klinischen Problem (klinische Fragestellung/rechtfertigende Indikation)

- Auswahl;des am besten geeigneten Kontrastmittels und seine optimale Verwendung entsprechend der Bildgebungstechnik und des klinischen Problems bei der Bildgebung des Bewegungsapparates

- Durchführung dynamischer Funktionsstudien der Wirbelsäule und der Gelenke 
- Grundlagen der Einstellungstechniken der Projektionsradiografie des Stammskeletts und der Extremitäten einschließlich aller Spezialeinstellungen

- Grundlagen der Ultraschalluntersuchung des Bewegungsapparates (Schnittführung, Gerätauswahl, Bildinterpretation)

- Beherrschung einfacher bildgesteuerter, perkutaner Biopsien, Drainagen und Schmerztherapien des Bewegungsapparates (Durchführung von komplexen bildgesteuerten Biopsien, Drainagen und Schmerztherapien (z. B. Ganglion-Blockaden) des Bewegungsapparates unter Aufsicht)

- Planung von CT-Untersuchungen des Bewegungsapparates bei individueller klinischer Situation und Fragestellung (individuelle Anwendung, keine standardisierte Protokollsituation z. B. Notfall) unter Beachtung des ALARA-Prinzips

- Planung von MRT-Untersuchungen des Bewegungsapparates bei individueller klinischer Situation und Fragestellung im Hinblick auf die die räumliche Auflösung, Wahl der Sequenzen und die mögliche Verwendung intravenöser Kontrastmittel

- Umfassende Kenntnisse über die Durchführung von Kontrastmittelinjektionen in Gelenke (z. B. Hüfte, Schulter und Handgelenk (z. B. MR-, CT-Arthrografie))

- Grundlegende Beherrschung der Technik der Diskografie, der Infiltration von Facettengelenken und perineuraler Strukturen (PRI) und der Vertebroplastie

- Sichere Anwendung von Nachbearbeitungsalgorithmen für Bildgebungsstudien im Muskel- und Skelettbereich, einschließlich multiplanarer Reformation (MPR), Maximalintensitätsprojektion (MIP), Minimalintensitätsprojektion (MinIP) und Fusion von Bildern oder Bildstudien

\section{II.3.2 Klinisch-radiologische Tätigkeiten}

- Komplette Kenntnisse zur Indikationsstellung diagnostischer und interventioneller Verfahren des Bewegungsapparates

- Umfassende Kenntnisse zur Moderation unterschiedlicher bildgebender Verfahren zur Beurteilung von Erkrankungen des Bewegungsapparates

- Umfassende Kommunikation mit dem Patienten zur Aufklärung, Beratung und Erlangung der Einverständniserklärung vor der diagnostischen Bildgebung oder vor interventionellen Verfahren des Bewegungsapparates sowie bei der Befunderläuterung (inkl. Angehöriger)

- Komplette Kenntnisse zur optimalen Wahl der Bildgebungsparameter für Röntgen- (ALARA-Prinzip/Strahlenschutzgesetzgebung), Ultraschall-, CT- (ALARA-Prinzip/Strahlenschutzgesetzgebung) und MRT-Untersuchungen des Bewegungsapparates

- Sicheres Erstellen von Bildgebungsprotokollen und Standardarbeitsanweisungen für CT- und MR-Untersuchungen der muskuloskelettalen Systems

- Überwachung und Unterweisung des technischen Personals zur Sicherstellung geeigneter Untersuchungsdokumente (hard- und soft-copy, DIN-Normierungen) des Bewegungsapparates

- Sichere Beherrschung der Qualitätskontrollvorgaben (DINNormen 6868 ff., Leitlinien der Bundesärztekammer in jeweils aktueller Fassung) von Bildgebungsuntersuchungen des Bewegungsapparates

- Komplette Beherrschung der Befundung und Beurteilung der relevanten bildgebenden Verfahren (Projektionsradiografie, CT, MR, Durchleuchtung, DAS und in Grundsätzen des Ultraschalls) des Bewegungsapparates unter Verwendung standardisierter Befundvorgaben (z. B. strukturierte Befundung, lexikalisierte Bildkriterien, Befundaufbau - DIN-Normen)

- Grundlegende Kenntnisse für die klinische Untersuchung des Bewegungsapparates (s. auch „rechtfertigende Indikation“/ Strahlenschutzgesetzgebung)

- Grundlegende Kenntnisse in der Befundung des Bewegungsapparates nach internationalen Standards (RECIST, WHO)

- Sicheres Erkennen von Notfällen

- Bereitschaft zur kollegialen und interdisziplinären Befunddiskussion

- Umfassende Erfahrungen, muskuloskelettale, interdisziplinäre Konferenzen (z. B. „Tumorboards“) und Falldiskussionen bei Erkrankungen des Bewegungsapparates durchzuführen (Nachweise)

\section{Lerninhalte Q2 Expertenwissen MSK-Radiologie}

\section{III.1 Allgemein}

- Komplette Kenntnisse der Anatomie aller für die Bildgebung des Bewegungsapparates relevanten Strukturen

- Umfassende Kenntnisse über normale Skelettvarianten, die Krankheiten imitieren können (z. B. Sesambeine oder „Schaltknochen“vv. Frakturen)

- Umfassendes Verständnis der Stufen der „Skelettentwicklung“"

- Grundlegende Kenntnisse der Embryologie des Skeletts

- Komplette Kenntnisse der klinischen Indikationen, Kontraindikationen und potenziellen Gefahren (z. B. Strahlenbelastung, Verfahrensbelastung) der bildgebenden Verfahren bei muskuloskelettalen Erkrankungen und bei Traumata

- Komplette Kenntnisse klinischer, insbesondere chirurgischer und internistischer, pathologischer und pathophysiologischer Zusammenhänge des Bewegungsapparates

- Komplette Kenntnisse der Bildgebungsmerkmale (lexikalisierte Befundkriterien) und klinischen Manifestationen von Erkrankungen und Traumata des Bewegungsapparates in der Projektionsradiografie, CT, MRT, Durchleuchtung (z. B. Arthrografie und Funktionsanalysen) und im Ultraschall

- Umfassende Kenntnisse der Differenzialdiagnosen, die für das klinische und bildgebende Erscheinungsbild von Erkrankungen oder Traumata des Bewegungsapparates relevant sind

- Umfassende Beherrschung der lexikalisierten Bildkriterien und deren differenzialdiagnostische Einordnung im Schriftbefund sowie umfassende Beherrschung klinisch etablierter Klassifikationen zur sicheren Zuordnung von Funktionsschaden, Therapiekonzepten und Outcome-Abschätzung 


\section{III.2 Wissen}

\section{III.2.1 Traumata - akut und Verlaufskontrolle}

- Umfassende Kenntnisse der Klassifizierungen von Frakturen und Luxationen, z. B. systematische Grundlagen der Outcome-, morphologisch- oder operativ-orientierten (z. B. AO- oder organspezifisch-operativ orientierte (z. B. Weber- oder Pauwels-)) oder Verletzungsmechanismus- bzw. funktionell-orientierten (z. B. Essex-Lopresti- oder Maisonneuve-Fraktur)) Klassifikationen

- Komplette Kenntnisse typischer und lexikalisierter Bildkriterien und umfassende klinische Kenntnisse von:

- Frakturen und Luxationen im Erwachsenenskelett (ab 14 Jahre/s. I.2.1.)

- derer Skelettentwicklung

- chondralen und osteochondralen Läsionen (z. B. Osteochondrose dissecans vs. flake-fracture)

- Konsolidierungsprozessen und Komplikationen bei Frakturen und Luxationen (z. B. verzögerte Konsolidierung, Pseudarthrose, avaskuläre Nekrose, Reflexdystrophie, komplexes und/ oder regionales Schmerzsyndrom, Myositis ossificans etc.)

\section{III.2.1.1 Frakturen und Luxationen im Einzelnen als Schwerpunktauflistung}

- Stressfrakturen, einschließlich Ermüdungs- und Insuffizienzbrüchen

- Schädel- und Gesichtsschädelfrakturen

- (Ausriss-) Avulsionsfrakturen und Insertionstendopathien

- pathologische, nichttraumatische Frakturen

- Frakturen der Wirbelsäule, einschließlich der Spondylolyse

- Frakturen und Luxationen der Schulter (z. B. sternoklavikuläre und akromioklavikuläre Luxationen, Klavikulafrakturen, Skapulafrakturen und Schulterluxation/,habituelle“ Instabilitäten)

- Frakturen und Luxationen der oberen Gliedmaßen (z. B. Humerusfrakturen, Ellenbogenfrakturen und -luxationen, proximale und distale Unterarmfrakturen und -luxationen, Handgelenkfrakturen und -luxationen und Handfrakturen und -luxationen)

- Frakturen und Luxationen des Beckens einschließlich Weichteilverletzungen

- Frakturen und Luxationen der unteren Extremitäten (z. B. Hüftfrakturen und -luxationen, Femurfrakturen, Tibia- und Fibulafrakturen (einschließlich des Sprunggelenks), Rückfußfrakturen, Tarsometatarsalfrakturen und -luxationen sowie Vorfußfrakturen und -luxationen)

- Verletzungen oder Gefügestörungen

- des Schultergelenks (z. B. der Rotatorenmanschette, des Glenoid-Labrum-Komplexes und der Bizepssehne)

- des Ellenbogengelenks

- des Handgelenks (z. B. des Discus triangularis und der interkarpalen Bänder)

- der Finger

- des Hüftgelenks (z. B. Labrum und FAl)

- des Knies (der Menisken, der Kreuz- und Kollateralbänder und der posterolateralen Ecke)
- des Sprunggelenks (z. B. Verletzungen und Gefügestörungen der Hauptsehnen, des Innen- und Außenbandapparates und des Tibiospringligaments)

- der Fußwurzel- und Zehengelenke

- Verletzungen der Muskeln und Muskelansätze, einschließlich deren Verlaufskontrolle

\section{III.2.2 Infektionen}

- Umfassende Kenntnisse typischer Bildkriterien und umfassende Kenntnisse klinischer Merkmale

- der akuten, subakuten und chronischen hämatogenen Osteomyelitis

- der posttraumatischen und postoperativen (Implantat-) Osteomyelitis

- der sicheren Methodenauswahl zur Erstdiagnostik und zum Therapiemonitoring (Röntgen, CT, Fistulografie, MR, Hybridverfahren, Grundkenntnisse nuklearmedizinischer Verfahren der Entzündungsdiagnostik: Tc-Szintigrafie, Leukozytenszintigrafie, PET- und SPECT-Verfahren)

- der Tuberkulose des Bewegungsapparates

- sonstiger spezifischer und seltener Infektionen (z. B. Lepra)

- von Weichteilinfektionen

- von HIV-assoziierten Infektionen

\section{III.2.3 Tumoren und tumor-like lesions}

- Umfassende Kenntnisse der typischen Bildkriterien, Prinzipien der Tumorcharakterisierung und des Stagings (z. B. klinisch, pathologisch-anatomisch/histologisch)

- von knochenbildenden Tumoren (z. B. Osteom, Osteoidosteom, Osteoblastom, Osteosarkom (typische Formen und Varianten))

- von knorpelbildenden Tumoren (z. B. Osteochondrom, Enchondrom, Chondroblastom, Chondromyxoid-Fibrom und Chondrosarkom (zentral und peripher))

- von fibrösen Tumoren (z. B. fibröser Kortikalisdefekt, nicht ossifizierendes Fibrom, fibröse Dysplasie, Fibrosarkom, malignes fibröses Histiozytom)

- von hämatopoetischen und retikuloendothelialen Tumoren (z. B. Riesenzelltumor, Langerhans-Zellhistiozytose, maligne Rundzelltumoren (Ewing-Sarkom, Lymphom und Leukosen), Myelom, Plasmozytom)

- einfacher und aneurysmatischer Knochenzysten

- von Metastasen

- von „dont't touch lesions“

- von Tumoren des Fettgewebes (z. B. Lipom und Liposarkom)

- von Tumoren neuronalen Ursprungs (z. B. Neurofibrom und Schwannom)

- von Tumoren vaskulären Ursprungs (z. B. Hämangiom)

- von Weichteilsarkomen

\section{III.2.4 Hämatologische Erkrankungen}

- Umfassende Kenntnisse der Bildkriterien und der klinischen Merkmale von Hämoglobinopathien (z. B. Sichelzellenanämie und Thalassämie)

- Umfassende Kenntnisse der Bildkriterien und der klinischen Merkmale der Myelofibrose 


\section{III.2.5 Metabolische, endokrine und toxische Erkrankungen}

- Umfassende Kenntnisse der Bildkriterien und der klinischen Merkmale

- der Rachitis und Osteomalazie

- des primären, sekundären und tertiären Hyperparathyreoidismus (einschließlich chronischer Niereninsuffizienz)

- der Osteoporose (einschließlich grundlegender Konzepte der Knochenbildgebung und Knochendichtemessung)

- der Fluorose und sonstiger Mineralosen

\section{III.2.6 Gelenke}

- Komplette Kenntnisse der Bildkriterien und umfassende Kenntnisse der klinischen Merkmale

- degenerativer Erkrankungen der Wirbelsäulen-, Bandscheiben- und Facettengelenke, der Becken- und peripherer Gelenke

- entzündlicher Erkrankungen (z. B. rheumatoide Arthritis, juvenile rheumatoide Arthritis, Spondylitis ankylosans, psoriatische Arthritis, enteropathische Arthropathien und infektiöse Arthritis (pyogen und tuberkulös), seronegative Arthritidien)

- von Kristallarthropathien (Pyrophosphat-Arthropathie, Hydroxyapatit-Ablagerungskrankheit und Gicht)

- von tumorartigen Gelenkveränderungen (z. B. Ganglion, synoviale Chondromatose, Morton-Neurom und pigmentierte villonoduläre Synovitis)

- bei Neuropathien (diabetischer Fuß, Charcot-Gelenke und Pseudo-Charcot-Gelenk (steroidinduziert))

- in der Endo- und Revisionsprothetik (Hüfte und Knie)

\section{III.2.7 Kongenitale und Entwicklungsstörungen}

- Grundlegende Kenntnisse der Bildkriterien und der klinischen Merkmale

- angeborener Erkrankungen der Wirbelsäule (z. B. Skoliose (kongenital und idiopathisch) und Dysraphien (inkomplett und komplett))

- angeborener Erkrankungen der Schulter (z. B. SprengelsDeformität)

- angeborener Hand- und Handgelenkstörungen (z. B. Madelung-Deformität (idiopathische und andere Ursachen))

- Erkrankungen der Hüfte (z. B. Hüftdysplasie, M. Perthes, Epiphysiolyse, femoroacetabuläres Impingement)

- der angeborenen Tarsalkoalition

- von Knochendysplasien (z. B. Dysmelien, Dysraphien, epi- und/ oder metaphysäre Dysplasien etc. - s. „Orpha-Klassifikation“)

- angeborener Knochenentwicklungsstörungen (z. B. Achondroplasie und osteogenesis imperfecta)

- angeborener Knochendichtestörungen (z. B. Osteopetrose, Melorheostose und Osteopoikilie)

- tumorähnlicher Läsionen (z. B. Exostosen/Osteochondrome und M. Ollier)

- der muskuloskelettalen Manifestationen der Neurofibromatose

\section{III.2.8 Verschiedenes}

- Umfassende Kenntnisse der Bildkriterien und umfassende Kenntnisse der klinischen Merkmale

- des M. Paget

- der Sarkoidose (inkl. M. Jüngling)

- der Varianten der hypertrophen Osteoarthropathien (seropositiv und-negativ)

- der transienten Osteoporose/-penie

- der Osteonekrosen (aseptische, traumatische etc.)

- der Weichteilverkalkungen/Weichteilossifikation

\section{III.3 KOMPETENZEN (Fertigkeiten und Fähigkeiten)}

\section{III.3.1 Technisch-klinische Anwendungen}

- Auswahl der geeignetsten Bildgebungsmethode des Bewegungsapparates abhängig vom klinischen Problem (klinische Fragestellung/rechtfertigende Indikation) - Umfassende und sichere Moderation der Methoden

- Auswahl des am besten geeigneten Kontrastmittels und seine optimale Verwendung entsprechend der Bildgebungstechnik und des klinischen Problems bei der Bildgebung des Bewegungsapparates - sichere Beherrschung aller Alternativverfahren (z. B. MR oder Spektral-CT)

- Durchführung dynamischer Funktionsstudien der Wirbelsäule und der Gelenke

- Grundlagen der Einstellungstechniken der Projektionsradiografie des Stammskeletts und der Extremitäten einschließlich aller Spezialeinstellungen

- Beherrschung der Ultraschalluntersuchungen des Bewegungsapparates (Schnittführung, Gerätauswahl, Bildinterpretation)

- Beherrschung bildgesteuerter, perkutaner Biopsien, Drainagen und Schmerztherapien des Bewegungsapparates

- Planung von CT-Untersuchung des Bewegungsapparates bei individueller klinischer Situation und Fragestellung (individuelle Anwendung, keine standardisierte Protokollsituation - z. B. Notfall) unter Beachtung des ALARA-Prinzips

- Planung von MRT-Untersuchung des Bewegungsapparates bei individueller klinischer Situation und Fragestellung im Hinblick auf die die räumliche Auflösung, Wahl der Sequenzen und die mögliche Verwendung intravenöser Kontrastmittel

- Komplette Kenntnisse und Fertigkeiten über bzw. für die Durchführung von Kontrastmittelinjektionen in Gelenke (z. B. Hüfte, Schulter und Handgelenk (z. B. MR-, CT-Arthrografie))

- Umfassende Beherrschung der Technik der Diskografie, der Infiltration von Facettengelenken und perineuraler Strukturen (PRI) und der Vertebroplastie

- Sichere Anwendung von Nachbearbeitungsalgorithmen für Bildgebungsstudien im Muskel- und Skelettbereich, einschließlich multiplanarer Reformation (MPR), Maximalintensitätsprojektion (MIP), Minimalintensitätsprojektion (MinIP) und Fusion von Bildern oder Bildstudien

- Umfassende Kenntnisse der nationalen und internationalen Literatur und deren sachdienliche Einordnung in individuelle Falldiskussionen 
- Umfassende Beherrschung gutachterlicher Befundzuordnungen hinsichtlich

- Schadensbild und

- dessen Kausalität

- Umfassende Vertretung des Fachgebiets der Bildgebenden Diagnostik gegenüber anderen klinischen Disziplinen und anderen Interessenvertretern (Gerichte, Staatsanwaltschaften, Behörden, Kostenträger etc.) inklusive individueller Einzelanwendung diagnostischer und/oder interventioneller Techniken bei komplexen klinischen Fragestellungen

\section{III.3.2 Klinisch-radiologische Tätigkeiten}

- Komplette Kenntnisse zur Indikationsstellung diagnostischer und interventioneller Verfahren des Bewegungsapparates

- Komplette Kenntnisse zur Moderation unterschiedlicher bildgebender Verfahren zur Beurteilung von Erkrankungen des Bewegungsapparates

- Komplette Kommunikation mit dem Patienten zur Aufklärung, Beratung und Erlangung der Einverständniserklärung vor der diagnostischen Bildgebung oder vor interventionellen Verfahren des Bewegungsapparates sowie bei der Befunderläuterung (inkl. Angehöriger)

- Komplette Kenntnisse zur optimalen Wahl der Bildgebungsparameter für Röntgen- (ALARA-Prinzip/Strahlenschutzgesetzgebung), Ultraschall-, CT- (ALARA-Prinzip/Strahlenschutzgesetzgebung) und MRT-Untersuchungen des Bewegungsapparates

- Sicheres Erstellen von Bildgebungsprotokollen und Standardarbeitsanweisungen für CT- und MR-Untersuchungen der muskuloskelettalen Systems
- Überwachung und Unterweisung des technischen Personals zur Sicherstellung geeigneter Untersuchungsdokumente (hard- und soft-copy, DIN-Normierungen) des Bewegungsapparates

- Sichere Beherrschung der Qualitätskontrollvorgaben (DINNormen 6868, Leitlinien der Bundesärztekammer in jeweils aktueller Fassung) von Bildgebungsuntersuchungen des Bewegungsapparates

- Komplette Beherrschung der Befundung und Beurteilung der relevanten bildgebenden Verfahren (Projektionsradiografie, CT, MR, Durchleuchtung, DSA und in Grundsätzen des Ultraschalls) des Bewegungsapparates unter Verwendung standardisierter Befundvorgaben (z. B. strukturierte Befundung, lexikalisierte Bildkriterien, Befundaufbau - DIN-Normen)

- Grundlegende Fähigkeiten und Fertigkeiten zur klinischen Untersuchung des Bewegungsapparates (s. auch „rechtfertigende Indikation“/Strahlenschutzgesetzgebung)

- Umfassende Kenntnisse in der Befundung des Bewegungsapparates nach internationalen Standards (RECIST, WHO)

- Sichere Erkennung von Notfällen

- Bereitschaft und umfassende Befähigung zur kollegialen und interdisziplinären Befunddiskussion und detaillierten Befundinterpretation

- Komplette Fähigkeiten, muskuloskelettale, interdisziplinäre Konferenzen (z. B. „Tumorboards“) und Falldiskussionen bei Erkrankungen des Bewegungsapparates durchzuführen (Nachweise) 